12. 1926. - SAdLer Wilfrid. Flore bactérienne du fromage de Kingston d'après les microphotographies. Trans. Roy. Socy. Can., $3^{\text {mes }}$ séries, vol. XX, section V, Ottawa.

13. 1906. - Harrison F.-C. Distribution des bactéries lactiques dans le caillé et le fromage du type Cheddar, avec 9 illustrations d'après les microphotographies. Trans. Roy. Soc. Can., $2^{\text {mer }}$ séries, 12, Sec..IV, 83-97.

\title{
RECHERCHES SUR LA VARIATION DE LA LACTATION DES VACHES GUERNESEY EN FONCTION DU TEMIPS
}

\author{
par W.L. GAINES.
}

\section{Introduction}

Au cours de l'année 1900, l'“ American Guernesey Cattle Club» adopta, aux Etats-Unis, un système de contrôle du rendement (Enregistrement avancé $=$ Advanced Registry $=$ A.R.), pour les vaches Guernesey, réalisant les conditions de rendement suivantes: La vache devait être de race pure, et enregistrée comme telle au "Herd Book "; elle devait avoir un minimum de rendement déterminé de matière grasse, au cours de 365 jours consécutifs. Ce minimum, pourles vaches de deux ans ou de moins de 2 ans était de 250,5 livres (114 kilogs), et augmentait dans une proportion donnée uniforme, avec chaque jour d'augmentation de l'âge, pour arriver à la quantité de 360 livres (164 kgs), à l'âge de cinq ans, à partir duquel, le minimum requis restait stationnaire. Depuis l'année 1923, le minimum exigé pour chaque âge a été augmenté.

Le propriétaire de la vache doit tenir une comptabilité exacte du poids de lait produit. Une fois par mois, non déterminée à l'avance, un contrôleur d'un Collège Agricole visite le troupeau, contrôle le rendement de lait et la teneur en matière grasse, pendant une période de deux jours. A l'aide de ces résultats, les rendements de lait et de matière grasse sont déterminés pour l'année. Depuis 1900 jusqu'à 1923, le Club publia les rendements enregistrés sous la forme indiquée par le tableau I; environ 15.000 rendements furent ainsi publiés. Il y a quelque différence entre le mode d'entretien des vaches dont le rendement est contrôlé, et le régime des vaches laitières ordinaires. En général, l’alimentation est très abondante; on prend des soins spéciaux, pour maintenir la sécrétion lactée au niveau le plus élevé possible, durant toute l'année; on retarde la fécondation, pendant un certain temps, parce que la vache non fécondée donne un rendement plus abondant, au cours de sa lactation. Cette méthode n'est pas la plus économique pour la production du lait: mais l'éleveur, en la circonstance, n'a en vue que l'élévation du rendement de sa vache, qui pourra servir de réclame à son élevage.

Ces rendements enregistrés, (A.R. Records), ont une importance indéniable pour l'étude biologique des rapports de la secrétion lactée 
avec l'évolution des rendements pendant la lactation; il est certainement avantageux de bien alimenter et bien soigner la vache, lorsqu'on veut donner à la glande mammaire la plus grande possibilité de manifester sa puissance de production. De même, le retard dans la fécondation, en certains cas, permet d'observer, durant toute l'année consécutive au vêlage, le fonctionnement de la glande mammaire, en l'absence d'une gestation eoncomitante, et de comparer ainsi la lactation des vaches pleines avec celle des vaches non fécondées.

Partant de ce point de vue biologique, nous avons consacré quelque temps, dans notre station expérimentale, à l'observation des rendements enregistrés de vaches Guernesey, publiés durant les années 1917 à 1922. Cet article est une brève revue de quelques-unes des données, mises en évidence par cette étude, qui furent publiées antérieurement (voir les références bibliographiques $1,2,3,5$ et 6 ).

\section{Equation de la courbe de lactation}

Pour étudier l'évolution de la secrétion lactée, pendant la lactation il y a avantage à exprimer la courbe de production par une équation. A cet effet, l'équation exponentielle, utilisée par Brody et ses collaborateurs (7), convient très bien. Cette équation peut être exprimée de la façon suivante :

$$
\mathrm{y}_{m}=\mathrm{Ae}^{-k t}(1)
$$

Dans cette équation, $\mathrm{y}_{m}$ est le rendement d'un mois déterminé; t, le nombre de mois depuis la date du vêlage, considérée en la circonstances comme point de départ de Ia lactation, et supposée avoir eu lieu au milieu du mois; A est une constante, représentant le degré initial de rendement; $e$ est la base des logarithmes neperiens; et $k$ est une constante qui représente le degré de regression du rendement par mois de lactation.

Cette équation (1) peut être considérée comme la courbe de variation du rendement en fonction du temps, si nous commettons l'erreur, parfaitement tolérable, de considérer que le rendement de chaque mois est rigoureusement proportionnel à la quantité observée par un contrôle laitier fait au milieu de ce mois. Les constantes limitantes, $\mathrm{A}$ et $\mathrm{k}$, expriment respectivement, $\mathrm{A}$, la faculté de secrétion, au début de la lactation et $\mathrm{k}$, la persistance de la lactation.

Dans les cas de gestation, le cours de la secrétion lactée, dans les derniers stades de cette gestation, présente une déviation appréciable par rapport à l'équation - type des vaches qui n'ont pas été saillies pendant l'année qui suit leur vèlage; il est indispensable d'avoir recours à un terme additionnel, pour exprimer cette déviation; l'équation alors devient la suivante :

$$
\mathrm{y}_{m}=\mathrm{Ae}^{-k t}-\mathrm{Be}^{k^{\prime}(t-c)}(
$$




\section{Tableau I}

Père Sir Ponto of Elmwood 25492

Père Katonah's Mack 17252

Mère Faithful of Elmwood 39350

Mère Flossie of Elmwood 35949

Père Rex of Eastside 12763 A.R.

Mère Dolly Varden of Eastside 20756

Eleveur M.E. Gifford, Sherman, N.Y.

Propriétaire George S. Love, Waukesha, Wis.

Née Déc. 1903. Vêlée Juin 4, 1920.

Saillie Avr. 4, 1921.

Conditions d'admission : 360,00 liv. Matière grasse.

Contrôlée par la Station du

Wisconsin.

3 Traites quotidiennes

Père Imp. Lord Mar V. 18961 A.R. 1920 Père Imp, Lord Mar 14359 A.R. Mère Imp. Countess I of Les Nouettes 36184

Mère Imp. Dora of the Vrangue VI. 36193

Père Imp. Galaxy Sequel 16904 A.R.

Mère Dora of the Vrangue

R.G.A.S. 5572 P.S.

Eleveur J.B. Haggin, Lexington, Ky.

Propriétaire R.M. Cooper Jr.

Wisacky S.C.

Née Nov. 15, 1913. Vêlée Déc. 16, 1919.

Saillie Oct. 8, 1920.

Conditions d'Admission : 360,00 liv. Matière grasse.

Contrôlée par la Station de la

6 Caroline du sud.

3.Traites quotidiennes.

Fév.

Mars.

Avr.

Mai.

Juin.

Août.

Sept.

Oct.

Not.
Record officiel de l'année, classe A Lait Matière grasse

\begin{tabular}{lrrr}
\multicolumn{1}{l}{$\begin{array}{l}1920 \\
\text { Juin 7, }\end{array}$} & Livres & $\%$ & Livres \\
Juillet, & $\mathbf{9 1 9 , 2}$ & 4,28 & 39,34 \\
Août, & 1184,5 & 4,34 & 54,88 \\
Septem., & 1179,6 & 4,40 & 51,90 \\
Octob. & 1236,1 & 4,60 & 56,80 \\
Novem. & 1181,6 & 4,47 & 52,82 \\
Décem. & 1212,0 & 4,51 & 54,66 \\
Jan. 1921 & 1205,4 & 4,75 & 57,26 \\
Fév. & 1077,8 & 5,05 & 54,43 \\
Mars. & 1115,8 & 4,81 & 53,57 \\
Avr. & 1109,1 & 4,78 & 63,01 \\
Mai. & 1113,5 & 5,45 & 60,69 \\
Juin 6, & 223,8 & 5,45 & 12,20 \\
\hline Total & 14020,3 & 4,66 & 653,25
\end{tabular}

Record officiel de l'année, classe A. Lait Matière grasse

Déc. 22

Livres \% Livres

Jan. $1920 \quad 1069,4 \quad 4,56 \quad 73,39$

$1244,1 \quad 4,96 \quad 61,71$

$1144,8 \quad 4,55$

52,09

$1022,9 \quad 4,52$

46,24

$892,0 \quad 4,98$

44,42

$656,8 \cdot 4,57$

30,02

Juillet.

$427,2 \quad 4,56$

19,48

$310,9 \quad 5,19 \quad 16,60$

$309,8 \quad 4,59 \quad 14,22$

288,9

5,40

15,60

Déc. 20.

223,7

4,67

10,45

$155,9 \quad 4,63$

7,22

$\overline{\text { Total }} \quad \overline{8792,6} \quad \overline{4,71} \quad \overline{414,11}$

Reproduction de deux Records (Rendements contrôlés et enregistrés) publiés par le " Guernsey Advanced Register " (Registre du Contrôle du Rendement de la Race Guernesey). 
Dans cette équation, $B \mathrm{e}^{k^{\prime}(t-a)}$ représente l'influence de la gestation sur la variation de la secrétion lactée; B et $k^{\prime}$ sont de nouvelles constantes ; c exprime le nombre de mois écoulés depuis le vêlage jusqu'à la fécondation; et (t-c) est la période qui s'étend depuis la fécondation jusqu'au moment du tarissement de la mamelle.

\section{Figure 1}

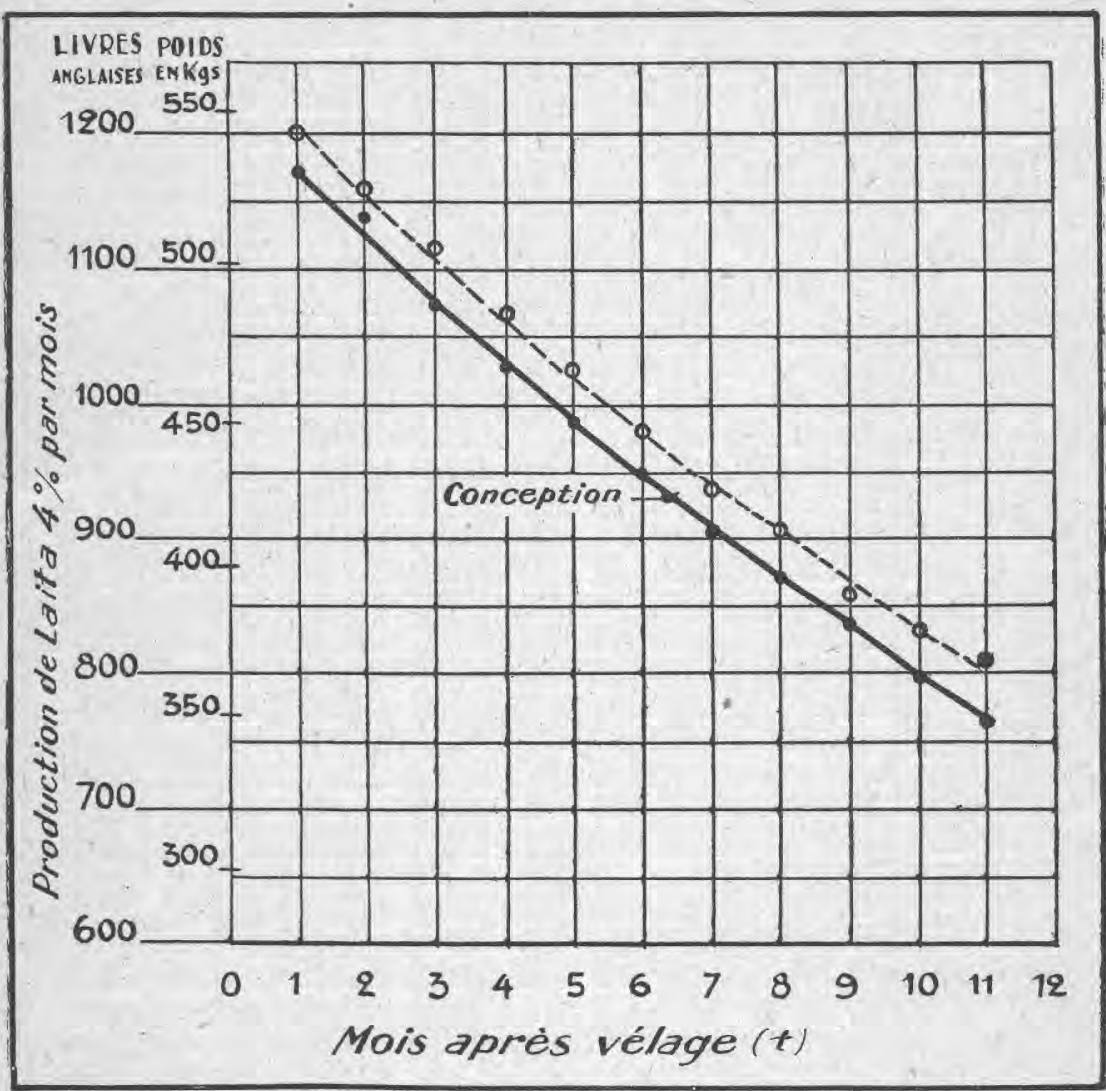

Variations mensuelles des rendements de 255 vaches non fécondées et de 575 vaches fécondées au cours du sixième mois de leur lactation.

\section{Détermination du rendement}

Le lait contient une quantité d'eau importante et variable. Si nous déterminons le rendement d'après le poids du lait produit, nous attribuons une importance égale à l'eau et aux constituants solides du lait. Si nous déterminons le rendement en poids de la matière grasse, nous négligeons totalement les autres constituants du lait. Ni l'un, ni l'autre de ces procédés ne donnent une estimation satisfaisante du rendement. 
Pour parer à ces objections, nous avons pensé que la valeur energétique brute du lait pouvait constituer une bonne mesure biologique du rendement réel de la mamelle. Pour faciliter les calculs, nous avons cherché uné formule qui puisse nous permettre de déterminer, en partant d'une quantité quelconque de lait à taux butyreux connu,

Figure 2

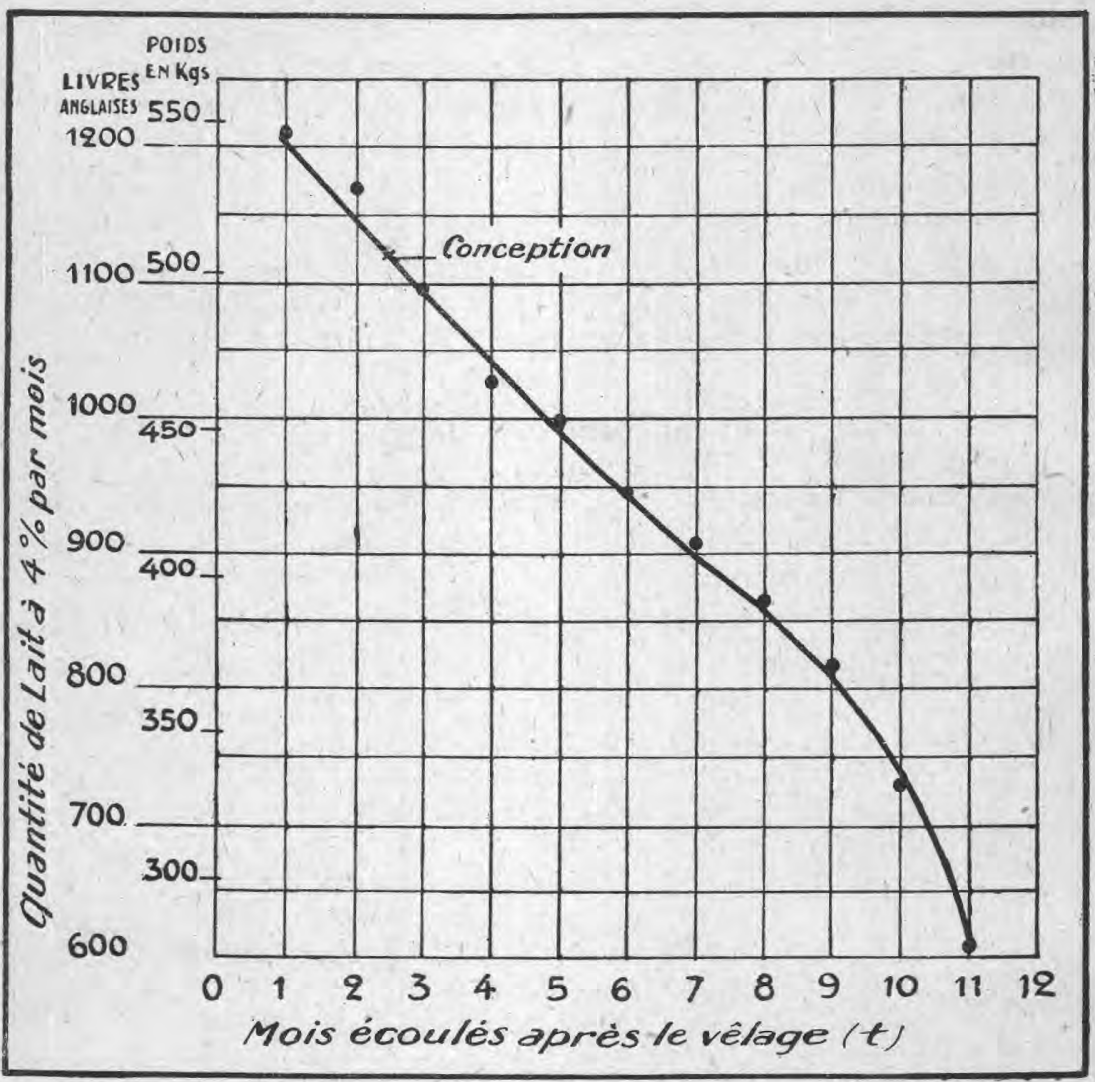

Variations mensuelles des rendements de 235 vaches, fécondées au cours du deuxième mois de leur lactation.

la quantité isodyname d'un lait à $4 \%$ de matière grasse. La formule ci-après nous a donné satisfaction

$$
\text { F.C.M }=0,4 \mathrm{M}+15 \mathrm{~F}
$$

Dans cette formule, F.C.M. représente la quantité cherchée de lait à $4 \%$ (Fat corrected Milk); M est la quantité de lait donné, et $\mathrm{F}$ représente la matière grasse totale contenue dans cette quantité M. 
Ainsi, par exemple, soit $100 \mathrm{~kg}$. de lait à $3.5 \%$ de matière grasse. $\mathrm{M}$ est ici égal à 100 , et $\mathrm{F}$ est égal à $\frac{100 \times 3,5}{100}=3^{\mathrm{k}} 5$

La formule donne F.C.M $=0,4 \times 100+15 \times 3.5=92 \mathrm{k} .5$.

Ceci nous dit que $100 \mathrm{k}$. de lait à $3.5 \%$ ont la même valeur énergétique que $92 \mathrm{k} .5$ du lait à $4 \%$, choisi comme étalon de mesure.

Les observations recueillies prouvent que la formule précédente donne des résultats qui sont extrêmement voisins de la valeur énergétique réelle, pour des laits de taux butyreux compris entre 2.5 et $7.5 \%$.

Il est d'ailleurs possible de transformer les valeurs énergétiques relatives en leurs valeurs absolues, à la condition de se souvenir qu'un kilog. de lait à $4 \%$ représente 750 calories. (Cf. OvERMan et SANMANN (9) et ANDERSEn (10). Estimé en F.C.M, le rendement de la vache repose sur une base dynamique. En somme, la vache étant considérée comme une machine, la courbe énergétique de la lactation représente le rendement réel de la machine.

\section{Courbes de lactation de groupes}

La Figure I représente les moyennes des rendements mensuels au cours d'une lactation de 255 vaches non fécondées, et de 575 vaches, fécondées pendant le sixième mois de leur lactation. Une équation du type 1 fut déterminée dans chaque cas. La figure 2 représente des données similaires pour 235 vaches, fécondées au cours du deuxième mois ; une équation du type 2 cadrant avec les données recueillies fut également recherchée avec succès; les résultats obtenus ont été les suivants :

\section{Epoque de la Conception}

Equation

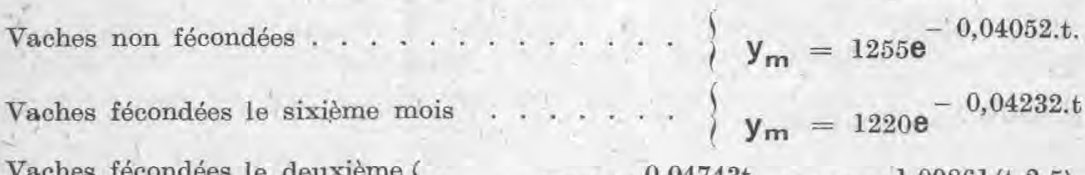

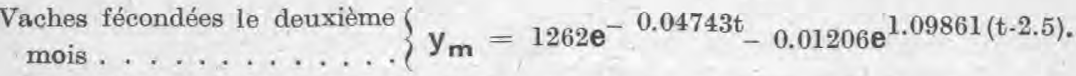

La figure 1 démontre que les équations du type 1 donnent des résultats en parfaite harmonie avec les chiffres réels fournis par la lactation des vaches non fécondées et celle des vaches fécondées le sixième mois de la lactation; d'où cette conclusion : La fécondation n'a pas d'influence appréciable sur le degré de la secrétion lactée, lorsqu'elle n'a pas lieu avant la fin du cinquième mois de lactation. Les équation et la figure 1 montrent que les vaches qui ne sont pas en gestation ont un degré initial de rendement plus élevé (1255) que les vaches fécondées (1220). Pour les premières, la regression mensuelle du rendement est de $4.052 \%$; pour les secondes, de $4,232 \%$. Il n'y a aucun motif 
apparent permettant d'attribuer la regression plus prononcée ou le degré initial de rendement moins élevé des vaches fécondées à l'influence de la conception. Le rendement d'une année des vaches non fécondées est plus élevé que celui des vaches fécondées, mais attribuer l'écart entre les rendements à l'influence de la gestation serait une erreur. Dans ce cas ei, la prudence s'impose dans l'interprétation des données statistiques.

La figure 2 démontre nettement qu'il y a une réduction très appréciable du rendement dans les derniers stades de la lactation pour les bêtes fécondées dans les premiers mois qui suivent le vêlage; la moyenne de regression pour chaque mois de progression de la lactation est de. $4,743 \%$ et ceci, indépendamment de l'influence de la gestation. Cette dernière cause provoque une réduction secondaire, qui se superpose à la regression principale, et qui est mesurée par l'expression soustraite de la première partie de la formule.

Calculée selon cette formule, la réduction totale du rendement pendant les cinq mois de la gestation serait de 2,5 livres $(1 \mathrm{~kg} .130)$ de lait à $4 \%$. Cette valeur est trop petite pour avoir une importance pratique; sa prise en considération est duldomaine de la théorie. Mais la réduction du rendement, pour toute la durée de la gestation ( 9,2 mois) est beaucoup plus importante; sa valeur est de 256 livres de lait à $4 \%$, soit $116 \mathrm{~kg}$. Cette réduction est-elle due au prélèvement dans le sang de la mère de constituants nutritifs par le fœtus; ou est-elle due à une hormone (chalone), dont la production est connexe fdu processus de la reproduction?

L'auteur admet la probabilité de la seconde hypothèse.

(A suivre).

\title{
CONTRIBUTION A L'ÉTUDE DE LA VALEUR PRATIQUE DES SIGNES LAITIERS ETEGBEURRIERS CHEZ LA VACHE
}

\author{
par A.-M. LEROY,
}

Chef de Travaux de Zootechnie à l'Institut National Agronomique

Di recteur de la Station volante d'expérimentation Zootechnique de l'Office Régional du Nord

$$
\text { (Fin) }
$$

\section{Comparaison des valeurs" mesurées aux grandeurs correspondantes [de la production flaitière et beurrière}

Afin d'arriver à un classement rationnel, d'après leur valeur pratique, des caractères précédemment énumérés, il était indiqué d'employer la méthode statistique des corrélations de Pearson. Cette méthode, encore assez peu pratiquée par les zootechniciens français, est utilisée couramment à l'étranger, et fournit des conclusions fort intéressantes par leur précision. Elle est particulièrement bien décrite dans le traité 